\title{
Performance evaluation of 3 serodiagnostic peptide epitopes and the derived multi-epitope peptide OvNMP-48 for detection of Onchocerca volvulus infection
}

\author{
Ole Lagatie $^{1}$ D $\cdot$ Ann Verheyen ${ }^{1} \cdot$ Erik Nijs $^{1} \cdot$ Linda Batsa Debrah $^{2} \cdot$ Yaw A. Debrah ${ }^{3} \cdot$ Lieven J. Stuyver $^{1}$
}

Received: 11 January 2019 / Accepted: 30 April 2019 / Published online: 14 May 2019

(C) The Author(s) 2019

\begin{abstract}
Current diagnostic tools to determine infection with the helminth parasite Onchocerca volvulus have limited performance characteristics. In previous studies, a proteome-wide screen was conducted to identify linear epitopes in this parasite's proteome, resulting in the discovery of 1110 antigenic peptide fragments. Here, we investigated three of these peptides using peptide ELISA's and evaluated their sensitivity and specificity. Epitope mapping was performed, and peptides were constructed that contained only the minimal epitope, flanked by a linker. Investigation of the performance of these minimal epitope peptides demonstrated that all three of them have a specificity (as defined by lack of response in non-helminth-infected individuals) of $100 \%$, low cross-reactivity $(5.6 \%, 5.6 \%$, and $9.3 \%$, respectively), but low sensitivity $(36.9 \%, 46.5 \%$, and $41.2 \%$, respectively). Some cross-reactivity was observed in samples from individuals infected with soil-transmitted helminths or Brugia malayi. Combining these three minimal epitopes in a single peptide, called OvNMP-48, resulted in a performance that exceeded the sum of the individual epitopes, with a sensitivity of $76.0 \%$, a specificity of $97.4 \%$, and a cross-reactivity of $11.1 \%$. Cross-reactivity was observed in some STH and Brugia malayi-infected individuals. This work opens the opportunity to start exploring how these novel linear epitope markers might become part of the $O$. volvulus diagnostic toolbox.
\end{abstract}

Keywords Onchocerca volvulus $\cdot$ River blindness $\cdot$ Onchocerciasis $\cdot$ Serology $\cdot$ Linear epitope

\section{Introduction}

Of the 20 infectious diseases listed on the World Health Organization (WHO) list of Neglected Tropical Diseases, eight

Section Editor: Sabine Specht

Electronic supplementary material The online version of this article (https://doi.org/10.1007/s00436-019-06345-3) contains supplementary material, which is available to authorized users.

Ole Lagatie

olagatie@its.jnj.com

Ann Verheyen

averheye@its.jnj.com

Erik Nijs

enijs@its.jnj.com

Linda Batsa Debrah

lindrousy@yahoo.com

Yaw A. Debrah

yadebrah@yahoo.com of them are caused by a helminth infection (Holmes 2014; Hotez et al. 2008; WHO 2018). One of them, onchocerciasis (or river blindness) is caused by infection with the filarial nematode Onchocerca volvulus. The majority of infected people
Lieven J. Stuyver

lstuyver@its.jnj.com

1 Global Public Health, Janssen R\&D, Turnhoutseweg 30, 2340 Beerse, Belgium

2 Kumasi Centre for Collaborative Research into Tropical medicine, Kwame Nkrumah University of Science and Technology, Kumasi, Ghana

3 Faculty of Allied Health Sciences, Kwame Nkrumah University of Science and Technology, Kumasi, Ghana 
live in Africa, where at least 120 million are at risk (Borup et al. 2003; Enk 2006). Current treatment programs are based on mass drug administration (MDA) of the microfilaricidal agent ivermectin (Mectizan, Merck) as no approved macrofilaricide drugs or vaccines are available. However, contraindications in areas co-endemic for loiasis and an inability to break transmission in some foci ask for a change in strategy including vaccination in order to control or eliminate onchocerciasis (Hotez et al. 2015; Makepeace et al. 2015).

Traditionally, Onchocerca infection is diagnosed by detection of microfilariae (mf) in skin biopsy samples (skin snips) (Taylor et al. 1989). As this requires an invasive procedure, several efforts have been undertaken to identify novel biomarkers that offer a less-invasive, specific and sensitive marker for infection with $O$. volvulus (Vlaminck et al. 2015). Only one of these, the rapid-format test for the detection of IgG4 antibodies to the parasitic antigen Ov-16, has been further developed and is commercially available (Golden et al. 2016). This serological test is currently being used for elimination mapping of onchocerciasis (Dieye et al. 2017; Lont et al. 2017; Richards et al. 2018).

Recently, two peptide-based serology markers, OvMP-1 and OvMP-23, were introduced. Although they demonstrate promising characteristics in the limited sample set that was evaluated with OvMP-23 having high sensitivity (92.7\%) and specificity (100\%), further evaluation of their clinical utility is warranted before they can be used in the field (Lagatie et al. 2018). Other approaches that have shown promise are the use of specific metabolites in serum or urine (Denery et al. 2010; Globisch et al. 2013; Lagatie et al. 2016b; Shirey et al. 2018) and the detection of parasitic microRNAs in the blood of infected individuals (Lagatie et al. 2016a; Quintana et al. 2015; Tritten et al. 2014a; Tritten et al. 2014b).

In our previous work, the entire $O$. volvulus proteome was screened for the presence of linear epitopes, and $1110 \mathrm{immu}-$ noreactive peptides have been identified in this proteome, of which 249 were considered immunodominant, and as such can also be considered serodiagnostic candidates (Lagatie et al. 2017). In the study presented here, we investigated three of these immunoreactive peptides. Immune signals observed on the peptide microarray were confirmed by peptide ELISA and the epitope was mapped.

\section{Materials and methods}

\section{Ethical statement}

All samples used in this study were de-identified before being provided, and usage of these samples for research purposes was approved by an ethical committee or Institutional Review Board (IRB). For samples collected in Ghana, a study was approved by the Committee on Human Research,
Publications and Ethics of the School of Medical Sciences of the Kwame Nkrumah University of Science and Technology, Kumasi, Ghana, and study subjects signed an informed consent form. For samples obtained from FR3, all were de-identified before they were provided to FR3, and usage of these samples for research purposes was approved by the Smith College Institutional Review Board (IRB). For samples collected in Belgium from healthy control donors, the Ethics Committee ["Commissie voor Medische Ethiek ZiekenhuisNetwerk Antwerpen (ZNA) and the Ethics committee University Hospital Antwerp] approved the protocol, and informed consent, which was signed by all subjects. For samples obtained from Tissue Solutions Ltd. (Glasgow, Scotland), Discovery Life Sciences, Inc. (Los Osos, USA), Bioreclamation IVT (Baltimore, USA), Mayo Clinic (Jacksonville, USA), and Universitas Indonesia or University of Ghent, written informed consent was obtained from all individuals, and all samples were decoded and deidentified before they were provided for research purposes.

\section{Study samples}

Plasma samples from $O$. volvulus-infected individuals were collected as part of a field study in Ghana. This study was undertaken in an onchocerciasis-endemic community located in Adansi South District along the Pra river basins in the Ashanti Region of Ghana. Physical examinations were performed to identify those subjects having palpable nodules. Skin snips (biopsies) were then taken in order to determine the microfilarial (mf) load in the skin (Debrah et al. 2015). Most subjects were participating in mass drug administration programs and had no or very low numbers $(<5 \mathrm{mf} / \mathrm{mg}$ skin) of microfilaria. Palpation is generally considered rather specific (estimated at $98 \%$ specific) but has somewhat lower sensitivity due to hidden nodules in deeper subcutaneous layers (Coffeng et al. 2013). No information was available on any other parasitic infections. A total of 93 nodule positive subjects that donated plasma samples were included (Table 1). Of those subjects, 63 were positive in the SD Bioline Ov16 IgG4 rapid test (i.e., 68\%), which is slightly lower than the reported sensitivity of $80 \%$ which was determined using ELISA (Golden et al. 2016).

Additionally, a second set of eight serum samples from O. volvulus-infected individuals, collected in Cameroon by Dr. Nutman, was obtained through the Filariasis Research Reagent Resource Center (FR3), Division of Microbiology and Infectious Diseases, NIAID, NIH. Information on $O$. volvulus infection (number of microfilaria/mg skin and number of palpable nodules) was provided by FR3, along with demographic information (Table 1). All infected individuals had at least two palpable nodules and $25 \mathrm{mf} / \mathrm{mg}$ skin (microfilaridermia) as determined by skin snip and were positive in the Ov16 IgG4 rapid test. 
For the non-helminth-infected control samples, demographic information is also provided in Table 1. A first healthy control sample set was composed of nine serum samples from individuals from Southern Africa, and was provided by Tissue Solutions Ltd. (Glasgow, Scotland). A second healthy control sample set was composed of 49 plasma samples from healthy individuals from Belgium. (Lagatie et al. 2014a; Lagatie et al. 2014b; Lagatie et al. 2014c; Stuyver et al. 2013; Van Loy et al. 2013). Furthermore, different sets of plasma samples were obtained from Discovery Life Sciences, Inc. (Los Osos, USA), Bioreclamation IVT (Baltimore, USA), FR3, or Mayo Clinic (Jacksonville, USA).

For the cross-reactivity panels (non-Onchocerca helminthinfected individuals), a set of ten samples from Wuchereria bancrofti-infected individuals was obtained through the Filariasis Research Reagent Resource Center (FR3), Division of Microbiology and Infectious Diseases, NIAID, and NIH. Samples were collected in Tahiti by Dr. Perolat (Institut Territorial de Recherches Médicales Louis Malardé, Tahiti, Polynésie Française) or Sri Lanka by an unknown collector, and information on W. bancrofti infection (number of microfilaria/mL) was provided by FR3, along with demographic information (Table 1). One set of 20 samples from individuals with Brugia malayi infection, collected in Central Sulawesi, Indonesia was kindly provided by Prof. Yazdanbakhsh (Leiden University Medical Center, Leiden, The Netherlands). A last sample set from 24 individuals infected with soil-transmitted helminths (STH) and/or Schistosoma mansoni, collected in Jimma, Ethiopia, was kindly provided by Prof. Levecke (Ghent University, Merelbeke, Belgium) (Table 1). STH infections included Ascaris lumbricoides, Trichuris trichiura, and hookworm (Necator americanus), either as single or mixed infected.

\section{Total IgG peptide ELISA}

Biotinylated synthetic peptides were synthesized by standard procedures and purchased from PEPperPRINT GmbH (Heidelberg, Germany). Peptides that only contain the minimal epitopes flanked at both termini by a small linker peptide (Val-Ser-Val) were designated OvNMP for $O$. volvulus nonmotif peptide (Table 3). For determination of peptide-specific serum antibody levels, peptide ELISA was developed and set up as described previously (Lagatie et al. 2017). For visualization purposes, all data points that had background-corrected absorbance below 0.01 were replaced by 0.005 to enable proper plotting on a logarithmic scale.

\section{Epitope mapping}

Permutation scans were carried out by PEPperPRINT GmbH (PEPperCHIP® Platform Technology, Heidelberg, Germany). In a permutation scan, the effect on binding of replacing each 
Table 2 Performance characteristics of the selected peptides in ELISA

\begin{tabular}{lllll}
\hline Peptide ID & Peptide sequence & $\begin{array}{l}\text { Sensitivity }(\%) \\
(n=50)\end{array}$ & $\begin{array}{l}\text { Specificity }(\%) \\
(n=38)\end{array}$ & $\begin{array}{l}\text { Median signal nodule } \\
\text { positives (+IQR) }\end{array}$ \\
\hline OVOC10067;2131 & TVEGGDNNGANFE & 24.0 & 100 & $0.02(0.00-0.08)$ \\
OVOC1858;283 & INRDANLNANSNPND & 35.0 & 100 & $0.06(0.02-0.15)$ \\
OVOC2814;121 & SDWDSEKDGKKKD & 44.0 & 100 & $0.08(0.02-0.17)$ \\
\hline
\end{tabular}

$I Q R$ interquartile range

of the amino acid residues by all amino-acids is analyzed, which requires the synthesis of $20 * \mathrm{x}$ spot peptides per starting peptide ( 20 amino-acids at $x$ positions, with $x$ the length of the peptide). Seven arrays were prepared, each containing the permutation analysis of the WT peptide and of 19 variants. Three thousand three hundred twenty different peptides were printed at least in duplicate and were framed by additional Influenza Hemagglutinin (HA) (YPYDVPDYAG) and polio (KEVPALTAVETGAT) control peptides (186 spots for each control). Each of the peptide arrays was incubated with 500fold diluted serum sample and stained with Goat anti-human IgG (Fc) DyLight680 (1:5000) and goat anti-human IgM ( $\mu$ chain) DyLight800 (1:5000). Arrays were scanned with LICOR Odyssey Imaging System, and fluorescence signals were used to calculate relative intensity compared to the native peptide. Consensus epitopes were derived from these analyses whereby the consensus epitope is defined as the region in the peptide for which a strong and consistent reduction in the signal is observed when a single amino acid is replaced by any other or a non-related amino acid.

\section{Statistical analysis}

For the minimal epitope peptides investigated in this study, ROC analysis was performed. Several sample sets from nonhelminth-infected individuals were used as control group. These include healthy controls from both Belgium and South Africa, HIV-infected or HCV-infected individuals from USA, dengue-infected individuals from Vietnam, and asthma patients from the USA. Two groups of $O$. volvulus-infected individuals were used as positive group: one from Cameroon and one from Ghana. ROC analysis was performed using all "non-helminth controls" vs. "all $O v$-positive samples" and cutoffs were determined as the point with maximal specificity, at the expense of a decrease of sensitivity. In the case of OvNMP-48, the cutoff was defined as the point with maximal Youden's index ((sensitivity + specificity)-1). Based on these cutoffs, sensitivity and specificity of each peptide ELISA were determined, as well as cross-reactivity with other helminths in samples with Brugia, Wuchereria, or STH infections. All analyses were performed using GraphPad Prism 7.

\section{Results}

\section{Confirmation of peptide microarray using peptide ELISA}

From the list of 1110 immunoreactive peptides, three were selected for further evaluation (results not shown) and peptide ELISA was developed. Immune reactivity against these peptides was determined in a random set of nodule-positive individuals $(n=50)$ and healthy controls $(n=38)$. Of importance, for both onchocerciasis patients and healthy controls, samples were different than those used in the peptide arrays (Lagatie et al. 2017). To be able to compare the performance of these peptides, an arbitrary cutoff was defined at 0.1 , and sensitivity and specificity were calculated for all peptides (Table 2). All three peptides were confirmed to be recognized by the antibodies in at least a subset of nodule-positive individuals.

\section{Determination of the minimal epitope}

To determine the key amino acids responsible for immune recognition, epitope mapping of these three peptides was performed (Table 3). Peptide microarrays covering full substitution scans of all four peptides, in which all amino acid positions were substituted by the 19 other amino acids, were

Table 3 Epitope mapping and sequence of peptides based on the minimal epitopes

\begin{tabular}{|c|c|c|c|c|}
\hline \multicolumn{3}{|l|}{ Epitope mapping } & \multicolumn{2}{|c|}{ Minimal epitope peptides } \\
\hline Peptide ID & Peptide sequence & Epitope & Peptide name & Sequence \\
\hline OVOC10067;2131 & TVEGGDNNGANFE & DNNGANFE & OvNMP-14 & VSV- DNNGANFE-VSV-Biotin \\
\hline OVOC1858;283 & INRDANLNANSNPND & NLNANSNPN & OvNMP-16 & VSV- NLNANSNPN-VSV-Biotin \\
\hline OVOC2814;121 & SDWDSEKDGKKKD & EKDGKK & OvNMP-18 & VSV- EKDGKK-VSV-Biotin \\
\hline
\end{tabular}


Table 4 Performance characteristics of $O$. volvulus minimal epitope containing peptides

\begin{tabular}{llllll}
\hline & Cutoff $^{1}$ & $\begin{array}{l}\text { Sensitivity }(\%) \\
(n=101)\end{array}$ & $\begin{array}{l}\text { Specificity }(\%) \\
(n=158)\end{array}$ & $\begin{array}{l}\text { Cross-reactivity } \\
(\%)(n=54)\end{array}$ & $\begin{array}{l}\text { Median signal nodule } \\
\text { positives (+IQR })\end{array}$ \\
\hline OvMP-1 & 0.045 & 100 & 98.7 & 72.0 & $3.39(2.67-3.62)$ \\
OvMP-23 & 0.11 & 92.7 & 100 & 6.0 & $0.64(0.30-1.26)$ \\
OvNMP-14 & $0.105^{*}$ & 36.9 & 100 & 5.6 & $0.06(0.02-0.20)$ \\
OvNMP-16 & $0.085^{*}$ & 46.5 & 100 & 5.6 & $0.07(0.02-0.15)$ \\
OvNMP-18 & $0.115^{*}$ & 41.2 & 100 & 9.3 & $0.08(0.01-0.27)$ \\
OvNMP-48 & 0.118 & 76.0 & 97.5 & 11.1 & $0.29(0.12-0.73)$ \\
\hline
\end{tabular}

${ }^{1}$ Background-corrected absorbance

$*$ Cutoff corresponding to $100 \%$ specificity

$I Q R$ interquartile range synthesized and analyzed using seven samples from nodulepositive individuals. For every single peptide and every sample, an amino acid plot was calculated (Fig. S1) and for each peptide, the consensus epitope is given in Table 3. Unfortunately, not all samples had sufficiently high reactivity for all peptides, resulting in the empty plots in Fig. S1. Although some minor differences exist between the different samples, the minimal epitopes of the peptides could be determined. For all three peptides, the epitopes do not share any similarity with previously described epitopes (Lagatie et al. 2017; Lagatie et al. 2018).

\section{Characterization of peptides with minimal epitopes}

Peptides were synthesized that only contain the minimal epitopes flanked at both termini by a small linker peptide (ValSer-Val). To determine the analytical performance of these peptides (sensitivity and specificity), reactivity of these peptides was assessed in sample sets from $O$. volvulus-infected individuals and different sets of non-helminth-infected individuals (Table 4). As a reference, also OvMP-1 and OvMP-23 are included in this table (Lagatie et al. 2018). For all three peptides, the specificity/sensitivity profile in ROC analysis allowed to define a cutoff that corresponded to $100 \%$ specificity, which makes them attractive markers for further evaluation.

As cross-reactivity with other helminth infections is an important issue in serological assays, we also investigated the reactivity of these peptides in sample sets from nonOnchocerca helminth-infected individuals (Table 4 and Fig. 1). The data demonstrate that, although all three peptides have $100 \%$ specificity, they display slight crossreactivity with other parasite infections $(5.6 \%, 5.6 \%$, and $9.3 \%$, respectively). Also, their sensitivity is too low to be useful as a standalone diagnostic marker $(36.9 \%, 46.5 \%$, and $41.2 \%$, respectively).

\section{Combination of different epitopes in one peptide}

As we demonstrated before, it is possible to combine different epitopes in one peptide to further optimize the performance of the assay (Lagatie et al. 2018). We therefore tested a peptide consisting of the three epitopes from OvNMP-14, OvNMP16, and OvNMP-18, separated by a small linker (VSVDNNGANFE-VSV-NLNANSNPN-VSV-EKDGKK-VSV, called OvNMP-48). Like the single epitope peptides, a
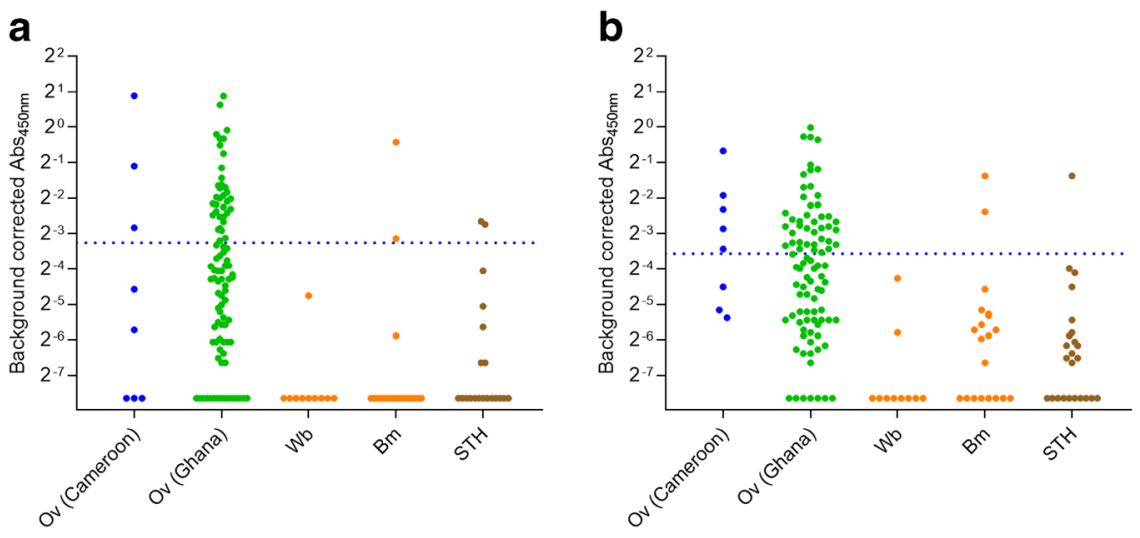

Fig. 1 Assessment of cross-reactivity against minimal epitope peptides. Immunoreactivity against OvNMP-14 (a), OvNMP-16 (b), and OvNMP18 (c) was determined in helminth-infected individuals. Dotted lines

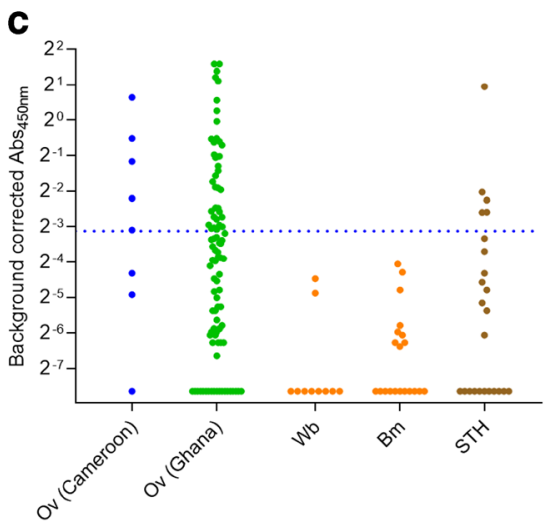

indicate the cutoff determined by ROC analysis. All samples used in this graph are described in Table 1 


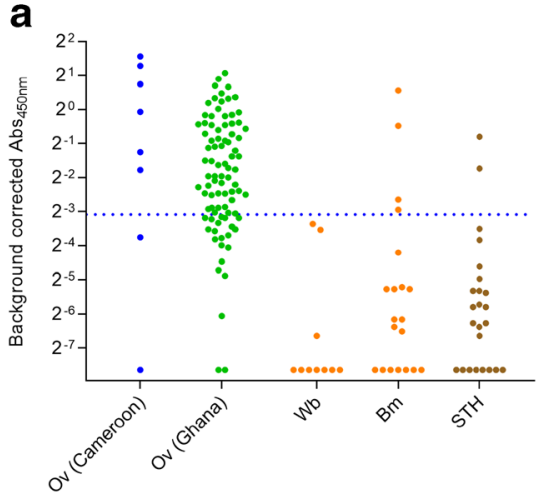

b

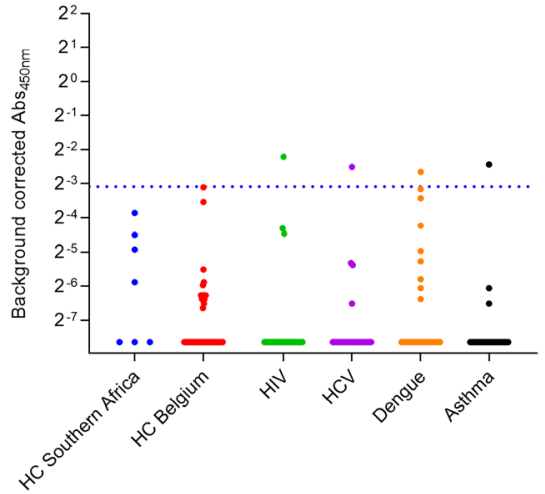

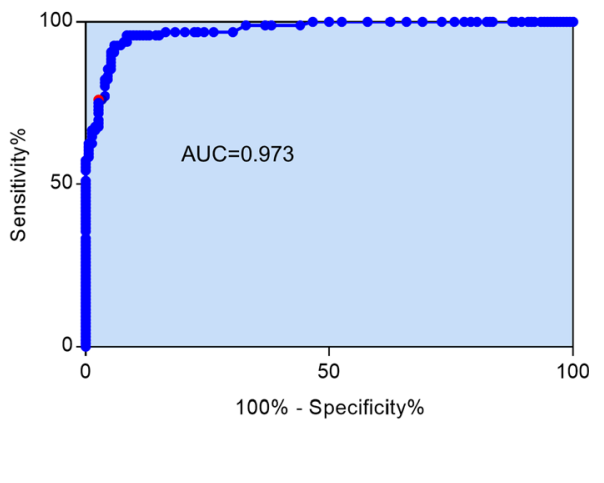

Fig. 2 Performance of a peptide with three epitopes in peptide ELISA. Immunoreactivity against OvNMP-48 was determined in helminthinfected individuals (a) and non-helminth-infected individuals (b). Dotted lines indicate the cutoff determined by ROC analysis. c ROC

specific cutoff was defined based on ROC analysis, resulting in a $97.4 \%$ specificity, $76.0 \%$ sensitivity, and a cross-reactivity of $11.1 \%$ (Table 4 . Cross-reactivity was not attributed to a specific helminth infection as 4 out of $20 \mathrm{~B}$. malayi and 2 out of $24 \mathrm{STH}$-infected individuals were found to be positive and none of the ten investigated samples from W. bancroftiinfected individuals was found to be positive. Some of the non-helminth-infected individuals also had an outlying positive response (Fig. 2).

\section{Discussion}

In this work, we have evaluated three immunoreactive $O$. volvulus peptides. We confirmed the peptide microarray data using peptide ELISA; epitope mapping was performed, and peptides were constructed that contained only the minimal epitope, flanked by a linker. Investigation of the performance of these minimal epitope peptides demonstrated that all three of them have a specificity of $100 \%$, low cross-reactivity $(<$ $10 \%)$, but rather low sensitivity $(<50 \%)$. Assessment of the performance of a combination of these three epitopes in one peptide, called OvNMP-48 showed that sensitivity could be further increased to $76.0 \%$, but with a small effect on specificity. It must be emphasized that for the control population, no information is available on any helminth infection and that therefore it cannot be excluded that some of these individuals had been exposed to helminths (and even $O$. volvulus), causing some reactivity against this peptide. The results demonstrate that combination of different linear epitopes, even if they have rather poor sensitivity, can result in a test that has a sufficiently good sensitivity/specificity profile to encourage further exploration and optimization of the epitopes and ultimately evaluation of its clinical utility. Besides the obvious increase in sensitivity, this result also demonstrates the possible drawback of combining multiple epitopes in one peptide analysis of onchocerciasis versus non-helminth infected individuals, with indication of the area under curve (AUC). All samples used in this graph are described in Table 1

as cross-reactivity of the different epitopes is now also reduced to a single result without information on which and how many epitopes are recognized by the antibodies in the sample. The use of multiple individual antigens in a classifier (immunosignature) might be better suited to overcome this issue as has been demonstrated before for Chagas disease (Zrein et al. 2018). An alternative strategy whereby multiple peptides are used in a mixture as capturing antigen was also shown to be a promising strategy to optimize sensitivity and specificity (Dubois et al. 2012). More work will be needed to investigate the performance of these linear epitopes as biomarker, either individually, as part of an immunosignature, or as combination in one peptide. Especially the assessment of cross-reactivity with other helminth species that are coendemic with $O$. volvulus, such as Loa loa, Mansonella, and Strongyloides, will be essential.

Acknowledgments We gratefully acknowledge the Filariasis Research Reagent Resource Center (FR3) at the Smith College, Northampton, USA for providing serum samples from $O$. volvulus-infected individuals under a contract from the National Institute of Allergy and Infectious Diseases, USA. We also acknowledge Prof. Maria Yazdanbakhsh for providing plasma samples of B. malayi from Indonesia and Prof. B. Levecke for providing plasma samples of STH-infected individuals from Ethiopia. We thank Janssen Biobank for logistic support, Will Colon for critically reading the manuscript, and Benny Baeten and Marc Engelen from Janssen Global Public Health for programmatic support.

Authors' contributions OL, AD, LBD and LJS designed the study. OL, $\mathrm{AV}$, and EN performed the research. OL and LJS performed the analysis of the data. OL and LJS wrote the manuscript. All authors read and approved the final manuscript.

\section{Compliance with ethical standards}

Ethical approval All procedures performed in studies involving human participants were in accordance with the ethical standards of the institutional and/or national research committee and with the 1964 Helsinki declaration and its later amendments or comparable ethical standards. 
Informed consent Informed consent was obtained from all individual participants included in the study, except for the samples obtained from FR3. These samples were de-identified before they were provided to FR3, and usage of these samples for research purposes was approved by the Smith College Institutional Review Board (IRB).

Conflict of interest OL, AV, EN, and LJS are current employees of Janssen Pharmaceutica NV, a Johnson and Johnson Company. OL and LJS own stock or stock options in that company.

Abbreviations O. volvulus, Onchocerca volvulus; mf, microfilaria; WHO, World Health Organization; MDA, Mass Drug Administration; ELISA, enzyme-linked immunosorbent assay; IRB, Institutional Review board; ROC, Receiver Operating Characteristic

Open Access This article is distributed under the terms of the Creative Commons Attribution 4.0 International License (http:// creativecommons.org/licenses/by/4.0/), which permits unrestricted use, distribution, and reproduction in any medium, provided you give appropriate credit to the original author(s) and the source, provide a link to the Creative Commons license, and indicate if changes were made.

\section{References}

Borup LH, Peters JS, Sartori CR (2003) Onchocerciasis (river blindness). Cutis 72(4):297-302

Coffeng LE, Pion SDS, O'Hanlon S, Cousens S, Abiose AO, Fischer PU, Remme JHF, Dadzie KY, Murdoch ME, de Vlas SJ, Basáñez MG, Stolk WA, Boussinesq M (2013) Onchocerciasis: the pre-control association between prevalence of palpable nodules and skin microfilariae. PLoS Negl Trop Dis 7(4):e2168. https://doi.org/10. 1371/journal.pntd.0002168

Debrah AY, Specht S, Klarmann-Schulz U, Batsa L, Mand S, MarfoDebrekyei Y, Fimmers R, Dubben B, Kwarteng A, OseiAtweneboana M, Boakye D, Ricchiuto A, Büttner M, Adjei O, Mackenzie CD, Hoerauf A (2015) Doxycycline leads to sterility and enhanced killing of female Onchocerca volvulus worms in an area with persistent Microfilaridermia after repeated ivermectin treatment: a randomized, placebo-controlled, double-blind trial. Clin Infect Dis : Off publ Infect Dis Soc Am 61(4):517-526. https://doi.org/10.1093/cid/civ363

Denery JR, Nunes AA, Hixon MS, Dickerson TJ, Janda KD (2010) Metabolomics-based discovery of diagnostic biomarkers for onchocerciasis. PLoS Negl Trop Dis 4(10):e834. https://doi.org/10.1371/ journal.pntd.0000834

Dieye Y, Storey HL, Barrett KL, Gerth-Guyette E, di Giorgio L, Golden A, Faulx D, Kalnoky M, Ndiaye MKN, Sy N, Mané M, Faye B, Sarr M, Dioukhane EM, Peck RB, Guinot P, de los Santos T (2017) Feasibility of utilizing the SD BIOLINE Onchocerciasis IgG4 rapid test in onchocerciasis surveillance in Senegal. PLoS Negl Trop Dis 11(10):e0005884. https://doi.org/10.1371/journal.pntd.0005884

Dubois ME, Hammarlund E, Slifka MK (2012) Optimization of peptidebased ELISA for serological diagnostics: a retrospective study of human monkeypox infection. Vector Borne Zoonotic Dis 12(5): 400-409. https://doi.org/10.1089/vbz.2011.0779

Enk CD (2006) Onchocerciasis-river blindness. Clin Dermatol 24(3): 176-180. https://doi.org/10.1016/j.clindermatol.2005.11.008

Globisch D, Moreno AY, Hixon MS, Nunes AAK, Denery JR, Specht S, Hoerauf A, Janda KD (2013) Onchocerca volvulus-neurotransmitter tyramine is a biomarker for river blindness. P Natl Acad Sci USA 110(11):4218-4223. https://doi.org/10.1073/pnas.1221969110
Golden A, Stevens EJ, Yokobe L, Faulx D, Kalnoky M, Peck R, Valdez M, Steel C, Karabou P, Banla M, Soboslay PT, Adade K, Tekle AH, Cama VA, Fischer PU, Nutman TB, Unnasch TR, de los Santos T, Domingo GJ (2016) A recombinant positive control for serology diagnostic tests supporting elimination of Onchocerca volvulus. PLoS Negl Trop Dis 10(1):e0004292. https://doi.org/10.1371/ journal.pntd.0004292

Holmes P, on behalf of the WHO Strategic and Advisory Group on Neglected Tropical Diseases (2014) Neglected tropical diseases in the post-2015 health agenda. Lancet 383(9931):1803. https://doi. org/10.1016/S0140-6736(14)60875-8

Hotez PJ, Brindley PJ, Bethony JM, King CH, Pearce EJ, Jacobson J (2008) Helminth infections: the great neglected tropical diseases. J Clin Invest 118(4):1311-1321. https://doi.org/10.1172/JCI34261

Hotez PJ, Bottazzi ME, Zhan B, Makepeace BL, Klei TR, Abraham D, Taylor DW, Lustigman S (2015) The onchocerciasis vaccine for Africa-TOVA-initiative. PLoS Negl Trop Dis 9(1):e0003422. https://doi.org/10.1371/journal.pntd.0003422

Lagatie O, Van Loy T, Tritsmans L, Stuyver LJ (2014a) Antibodies reacting with JCPyV_VP2_167-15mer as a novel serological marker for JC polyomavirus infection. Virol J 11:174. https://doi.org/10. 1186/1743-422X-11-174

Lagatie O, Van Loy T, Tritsmans L, Stuyver LJ (2014b) Circulating human microRNAs are not linked to JC polyomavirus serology or urinary viral load in healthy subjects. Virol J 11(1):41. https://doi. org/10.1186/1743-422X-11-41

Lagatie O, Van Loy T, Tritsmans L, Stuyver LJ (2014c) Viral miRNAs in plasma and urine divulge JC polyomavirus infection. Virol J 11:158. https://doi.org/10.1186/1743-422X-11-158

Lagatie O, Batsa Debrah L, Debrah A, Stuyver L (2016a) Plasma-derived parasitic microRNAs have insufficient concentrations to be used as diagnostic biomarker for detection of Onchocerca volvulus infection or treatment monitoring. PLoS neglected tropical diseases Submitted

Lagatie O, Njumbe Ediage E, Batsa Debrah L, Diels L, Nolten C, Vinken P, Debrah A, Dillen L, Silber S, Stuyver LJ (2016b) Evaluation of the diagnostic potential of urinary N-Acetyltyramine-O,beta-glucuronide (NATOG) as diagnostic biomarker for Onchocerca volvulus infection. Parasit Vectors 9(1):302. https://doi.org/10.1186/s13071016-1582-6

Lagatie O, Van Dorst B, Stuyver LJ (2017) Identification of three immunodominant motifs with atypical isotype profile scattered over the Onchocerca volvulus proteome. PLoS Negl Trop Dis 11(1): e0005330. https://doi.org/10.1371/journal.pntd.0005330

Lagatie O, Verheyen A, Nijs E, van Dorst B, Batsa Debrah L, Debrah A, Supali T, Sartono E, Stuyver LJ (2018) Evaluation of the diagnostic performance of Onchocerca volvulus linear epitopes in a peptide enzyme-linked immunosorbent assay. Am J Trop Med Hyg 98(3): 779-785. https://doi.org/10.4269/ajtmh.17-0756

Lont YL, Coffeng LE, de Vlas SJ, Golden A, de los Santos T, Domingo GJ, Stolk WA (2017) Modelling anti-Ov16 IgG4 antibody prevalence as an indicator for evaluation and decision making in onchocerciasis elimination programmes. PLoS Negl Trop Dis 11(1): e0005314. https://doi.org/10.1371/journal.pntd.0005314

Makepeace BL, Babayan SA, Lustigman S, Taylor DW (2015) The case for vaccine development in the strategy to eradicate river blindness (onchocerciasis) from Africa. Expert Review Vaccines 14(9):11631165. https://doi.org/10.1586/14760584.2015.1059281

Quintana JF, Makepeace BL, Babayan SA, Ivens A, Pfarr KM, Blaxter M, Debrah A, Wanji S, Ngangyung HF, Bah GS, Tanya VN, Taylor DW, Hoerauf A, Buck AH (2015) Extracellular Onchocerca-derived small RNAs in host nodules and blood. Parasit Vectors 8:58. https:// doi.org/10.1186/s13071-015-0656-1

Richards FO et al (2018) Operational performance of the Onchocerca volvulus "OEPA" Ov16 ELISA serological assay in mapping, guiding decisions to stop mass drug administration, and posttreatment 
surveillance surveys. Am J Trop Med Hyg 99(3):749-752. https:// doi.org/10.4269/ajtmh.18-0341

Shirey R, Globisch D, Eubanks LM, Hixon MS, Janda KD (2018) Noninvasive urine biomarker lateral flow immunoassay for monitoring active onchocerciasis. ACS Infect Dis 4:1423-1431. https://doi.org/ 10.1021/acsinfecdis. 8 b00163

Stuyver LJ, Verbeke T, Van Loy T, Van Gulck E, Tritsmans L (2013) An antibody response to human polyomavirus 15-mer peptides is highly abundant in healthy human subjects. Virol J 10:192. https://doi.org/ 10.1186/1743-422X-10-192

Taylor HR, Munoz B, Keyvan-Larijani E, Greene BM (1989) Reliability of detection of microfilariae in skin snips in the diagnosis of onchocerciasis. Am J Trop Med Hyg 41(4):467-471

Tritten L, Burkman E, Moorhead A, Satti M, Geary J, Mackenzie C, Geary T (2014a) Detection of circulating parasite-derived microRNAs in filarial infections. PLoS Negl Trop Dis 8(7):e2971. https://doi.org/10.1371/journal.pntd.0002971

Tritten L, O'Neill M, Nutting C, Wanji S, Njouendoui A, Fombad F, Kengne-Ouaffo J, Mackenzie C, Geary T (2014b) Loa loa and Onchocerca ochengi miRNAs detected in host circulation. Mol Biochem Parasitol 198(1):14-17. https://doi.org/10.1016/j. molbiopara.2014.11.001

Van Loy T, Thys K, Tritsmans L, Stuyver LJ (2013) Quasispecies analysis of JC virus DNA present in urine of healthy subjects. PLoS One 8(8):e70950. https://doi.org/10.1371/journal.pone.0070950
Vlaminck J, Fischer PU, Weil GJ (2015) Diagnostic tools for onchocerciasis elimination programs. Trends Parasitol 31(11):571-582. https://doi.org/10.1016/j.pt.2015.06.007

WHO (2018) Progress report on the elimination of human onchocerciasis, 2017-2018. In: Cantey P (ed) Releve epidemiologique hebdomadaire/section d'hygiene du secretariat de la Societe des nations $=$ weekly epidemiological record/health section of the secretariat of the league of nations. Vol 93. WHO, p 633-48

Zrein M, Granjon E, Gueyffier L, Caillaudeau J, Liehl P, Pottel H, Cardoso CS, Oliveira CDL, de Oliveira LC, Lee TH, Ferreira AM, Ribeiro ALP, Busch MP, Sabino EC (2018) A novel antibody surrogate biomarker to monitor parasite persistence in Trypanosoma cruzi-infected patients. PLoS Negl Trop Dis 12(2):e0006226. https://doi.org/10.1371/journal.pntd.0006226

Publisher's note Springer Nature remains neutral with regard to jurisdictional claims in published maps and institutional affiliations. 